\title{
Precise Analysis of Spectral Reflectance Properties of Cosmetic Foundation
}

\author{
Yusuke Moriuchi, Shoji Tominaga, and Takahiko Horiuchi \\ Graduate School of Advanced Integration Science, Chiba University, \\ 1-33, Yayoi-cho, Inage-ku, Chiba 263-8522, Japan
}

\begin{abstract}
The present paper describes the detailed analysis of the spectral reflection properties of skin surface with make-up foundation, based on two approaches of a physical model using the Cook-Torrance model and a statistical approach using the PCA. First, we show how the surface-spectral reflectances changed with the observation conditions of light incidence and viewing, and also the material compositions. Second, the Cook-Torrance model is used for describing the complicated reflectance curves by a small number of parameters, and rendering images of $3 \mathrm{D}$ object surfaces. Third, the PCA method is presented the observed spectral reflectances analysis. The PCA shows that all skin surfaces have the property of the standard dichromatic reflection, so that the observed reflectances are represented by two components of the diffuse reflectance and a constant reflectance. The spectral estimation is then reduced to a simple computation using the diffuse reflectance, some principal components, and the weighting coefficients. Finally, the feasibility of the two methods is examined in experiments. The PCA method performs reliable spectral reflectance estimation for the skin surface from a global point of view, compared with the model-based method.
\end{abstract}

Keywords: Spectral reflectance analysis, cosmetic foundation, color reproduction, image rendering.

\section{Introduction}

Foundation has various purposes. Basically, foundation makes skin color and skin texture appears more even. Moreover, it can be used to cover up blemishes and other imperfections, and reduce wrinkles. The essential role is to improve the appearance of skin surfaces. Therefore it is important to evaluate the change of skin color by foundation. However, there was not enough scientific discussion on the spectral analysis of foundation material and skin with make-up foundations [1]. In a previous report [2], we discussed the problem of analyzing the reflectance properties of skin surface with make-up foundation. We presented a new approach based on the principal-component analysis (PCA), useful for describing the measured spectral reflectances, and showed the possibility of estimating the reflectance under any lighting and viewing conditions.

The present paper describes the detailed analysis of the spectral reflection properties of skin surface with make-up foundation by using two approaches based on a 
physical model approach and a statistical approach. Foundations with different material compositions are painted on a bio-skin. Light reflected from the skin surface is measured using a gonio-spectrophotometer.

First, we show how appearances of the surface, including specularity, gloss, and matte appearance, change with the observation conditions of light incidence and viewing, and also the material compositions. Second, we use the Cook-Torrance model as a physical reflection model for describing the three-dimensional (3D) reflection properties of the skin surface with foundation. This model is effective for image rendering of $3 \mathrm{D}$ object surfaces. Third, we use the PCA as a statistical approach for analyzing the reflection properties. The PCA is effective for statistical analysis of the complicated spectral curves of the skin surface reflectance. We present an improved algorithm for synthesizing the spectral reflectance. Finally, the feasibility of both approaches is examined in experiments from the point of view of spectral reflectance analysis and color image rendering.

\section{Foundation Samples and Reflectance Measurements}

Although the make-up foundation is composed of different materials such as mica, talc, nylon, titanium, and oil, the two materials of mica and talc are the important components which affect the appearance of skin surface painted with the foundation. So many foundations were made by changing the quantity and the ratio of two materials. For instance, the combination ratio of mica $(M)$ and talc $(T)$ was changed as $(M=0, T=60)$, $(\mathrm{M}=10, \mathrm{~T}=50), \ldots,(\mathrm{M}=60, \mathrm{~T}=0)$, the ratio of mica was changed with a constant $\mathrm{T}$ as $(\mathrm{M}=0, \mathrm{~T}=40),(\mathrm{M}=10, \mathrm{~T}=40), \ldots,(\mathrm{M}=40, \mathrm{~T}=40)$, and the size of mica was also changed in the present study. Table 1 shows typical foundation samples used for spectral reflectance analysis. Powder foundations with the above compositions were painted on a flat bio-skin surface with the fingers. The bio-skin is made of urethane which looks like human skin. Figure 1 shows a board sample of bio-skin with foundation. The foundation layer is very thin as 5-10 microns in thickness on the skin.

Table 1. Foundation samples with different composition of mica and talc

\begin{tabular}{ccccccc}
\hline Samples & IKD-0 & IKD-10 & IKD-20 & IKD-40 & IKD-54 & IKD-59 \\
\hline Mica & 0 & 10 & 20 & 40 & 54 & 59 \\
\hline Talc & 59 & 49 & 39 & 19 & 5 & 0 \\
\hline
\end{tabular}

A gonio-spectrophotometer is used for observing surface-spectral reflections of the skin surface with foundations under different lighting and viewing conditions. This instrument has two degrees of freedom on the light source position and the sensor position as shown in Fig. 2, although in the real system, the sensor position is fixed, and both light source and sample object can rotate. The ratio of the spectral radiance from the sample to the one from the reference white diffuser, called the spectral radiance factor, is output as spectral reflectance. The spectral reflectances of all samples were measured at 13 incidence angles of $0,5,10, \ldots, 60$ degrees and 81 viewing angles of $-80,-78, \ldots,-2,0,2, \ldots, 78,80$ degrees. 


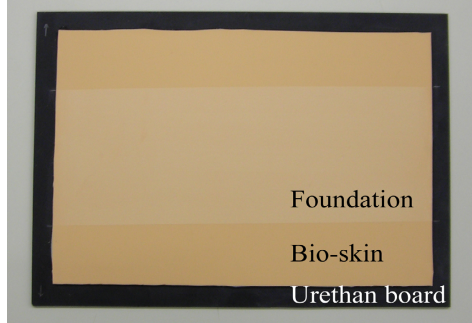

Fig. 1. Sample of bio-skin with foundation

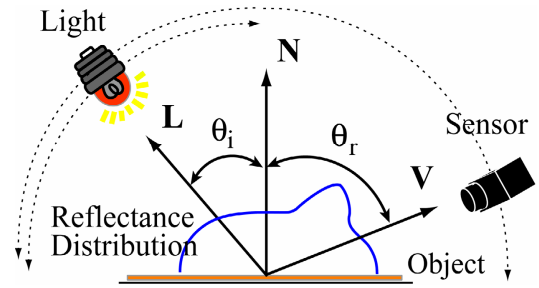

Fig. 2. Measuring system of surface reflectance

Figure 3(a) shows a 3D perspective view of spectral radiance factors measured from the bio-skin itself and the skin with a foundation sample IKD-54 at the incidence angle of 20 degrees. This figure suggests how the foundation changes effectively the spectral reflectance of the skin surface. In Fig. 3(a), solid mesh and broken mesh indicate the spectral radiance factors from bio-skin and IKD-54 itself, respectively, where the spectral curves are depicted as a function of viewing angle.

The spectral reflectance depends not only on the viewing angle, but also on the incidence angle. In order to make this point clear, we average the radiance factors on wavelength in the visible range. Figure 3(b) depicts a set of the average curves at different incidence angles as a function of viewing angle for both bio-skin and IKD-54.

A comparison between solid curves and broken curves in Fig. 3 suggests several typical features of skin surface reflectance with foundation as follows:

(1) Reflectance hump at around the vertical viewing angle,

(2) Back-scattering at around -70 degrees, and

(3) Specular reflectance with increasing viewing angle.

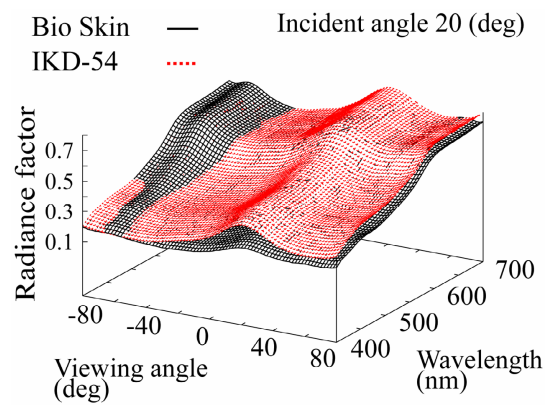

(a)

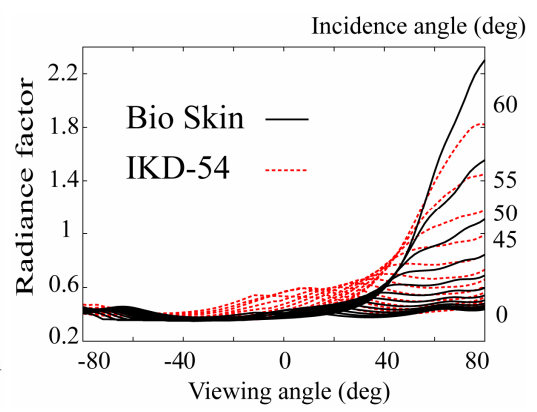

(b)

Fig. 3. Reflectance measurements from a sample IKD-54 and bio-skin. (a) 3D view of spectral reflectances at $\theta_{i}=20$, (b) Average reflectances as a function of viewing angle.

Moreover we have investigated how the surface reflectance depends on the material composition of foundation. Figure 4 shows the average reflectances for three cases among difference material compositions. As a result, we find the following two basic properties: 


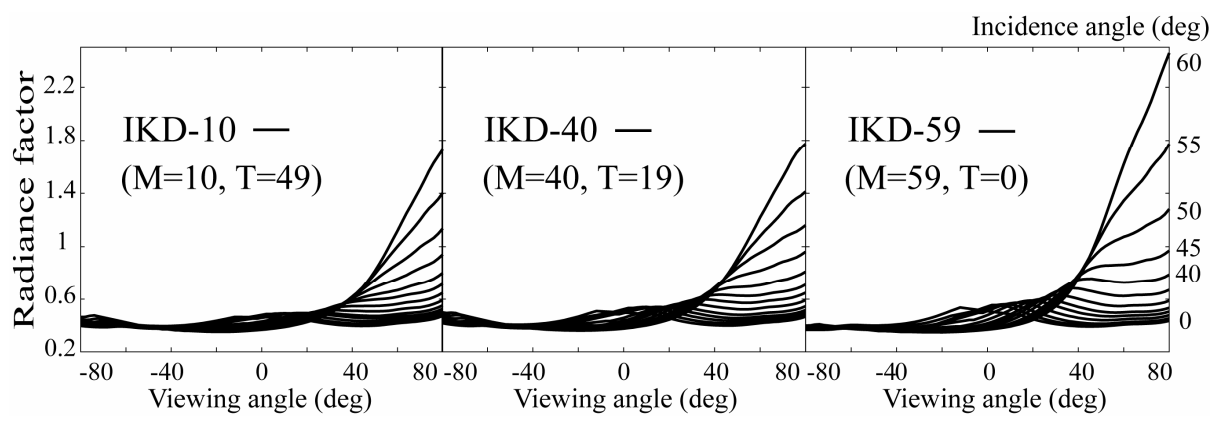

Fig. 4. Reflectance measurements from different make-up foundations

(1) When the quantity of mica increases, the whole reflectance of skin surface increases at all angles of incidence and viewing.

(2) When the quantity of talc increases, the surface reflectance decreases at large viewing angles, but increases at matte regions.

\section{Model-Based Analysis of Spectral Reflectance}

In the field of computer graphics and vision, the Phong model [3] and the CookTorrance model [4] are known as a 3D reflection model used for describing light reflection of an object surface. The former model is convenient for inhomogeneous dielectric object like plastics, although the mathematical expression is simple, and the number of model parameters is small. The latter model is a physically precise model which is available for both dielectrics and metals. In this paper, we analyze the spectral reflectances of the skin surface based on the Cook-Torrance model.

The Cook-Torrance model can be written in terms of the spectral radiance factor as

$$
Y(\lambda)=S(\lambda)+\beta \frac{D(\varphi, \gamma) G(\mathbf{N}, \mathbf{V}, \mathbf{L}) F\left(\theta_{Q}, n\right)}{\cos \theta_{i} \cos \theta_{r}},
$$

where the first and second terms represent, respectively, the diffuse and specular reflection components. $\beta$ is the specular reflection coefficient. A specular surface is assumed to be an isotropic collection of planar microscopic facets by Torrance and Sparrow [5]. The area of each microfacet is much smaller than the pixel size of an image. Note that the surface normal vector $\mathbf{N}$ represents the normal vector of a macroscopic surface. Let $\mathbf{Q}$ be the vector bisector of an $\mathbf{L}$ and $\mathbf{V}$ vector pair, that is, the normal vector of a microfacet. The symbol $\theta_{i}$ is the incidence angle, $\theta_{r}$ is the viewing angle, $\varphi$ is the angle between $\mathbf{N}$ and $\mathbf{Q}$, and $\theta_{Q}$ is the angle between $\mathbf{L}$ and $\mathbf{Q}$.

The specular reflection component consists of several terms: $D$ is the distribution function of the microfacet orientation, and $F$ represents the Fresnel spectral reflectance [6] of the microfacets. $G$ is the geometrical attenuation factor. $D$ is assumed as a Gaussian distribution function with rotational symmetry about the surface normal $\mathbf{N}$ as $D(\varphi, \gamma)=\exp \left\{-\log (2) \varphi^{2} / \gamma^{2}\right\}$, where the parameter $\gamma$ is a constant that represents surface roughness. The Fresnel reflectance $F$ is described as a nonlinear function with the parameter of the refractive index $n$. 
The unknown parameters in this model are the coefficient $\beta$, the roughness $\gamma$ and the refractive index $n$. The reflection model is fitted to the measured spectral radiance factors by the method of least squares. In the fitting computation, we used the average radiance factors on wavelength in the visible range. We determine the optimal parameters to minimize the squared sum of the fitting error

$$
e=\min \sum_{\theta_{i}, \theta_{r}}\left\{\overline{Y(\lambda)}-\overline{S(\lambda)}-\beta \frac{D(\varphi, \gamma) G(\mathbf{N}, \mathbf{V}, \mathbf{L}) F\left(\theta_{Q}, n\right)}{\cos \theta_{i} \cos \theta_{r}}\right\}^{2},
$$

where $\overline{Y(\lambda)}$ and $\overline{S(\lambda)}$ are the average values of the measured and diffuse spectral reference factors, respectively. The diffuse reflectance $S(\lambda)$ is chosen as a minimum of the measured spectral reflectance factors. The above error minimization is done over all angles of $\theta_{i}$ and $\theta_{r}$. For simplicity of the fitting computation, we determine the refractive index $n$ to 1.90 because the skin surface with foundation is considered as inhomogeneous dielectric.

Figure 5(b) shows the results of model fitting to the sample IKD-54 shown in Fig. 3, where solid curves indicate the fitted reflectances, and a broken curve indicates the original measurements. Figure 5(a) shows the fitting results for spectral reflectances at the incidence angle of 20 degrees. The model parameters were estimated as $\beta=0.74$ and $\gamma=0.20$. The squared error was $e=4.97$. These figures suggest that the model describes the surface-spectral reflectances at the low range of viewing angle with relatively good accuracy. However the fitting error tends to increase with the viewing angle.

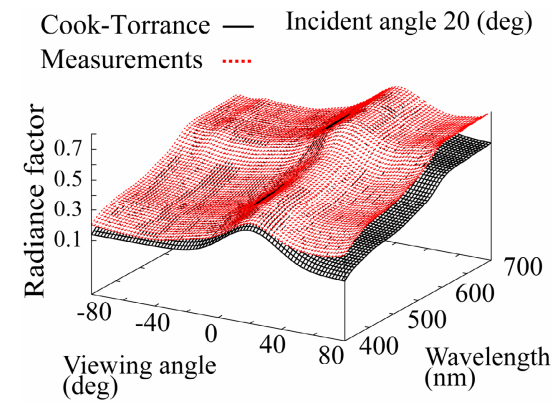

(a)

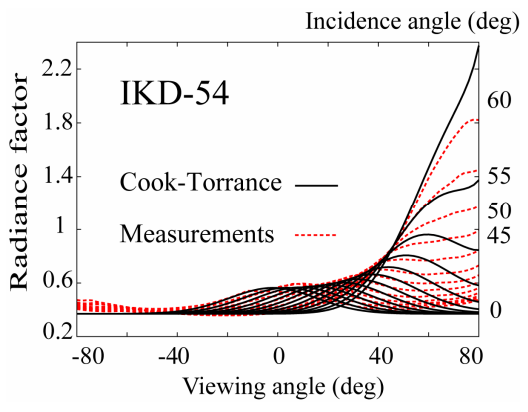

(b)

Fig. 5. Fitting results of the Cook-Torrance model to IKD-54. (a) $3 \mathrm{D}$ view of spectral reflectances at $\theta_{i}=20$, (b) Average reflectances as a function of viewing angle.

We have repeated the same fitting experiment of the model to many skin samples with different material compositions for foundation. Then a relationship between the material compositions and the model parameters was found as follows:

(1) As the quantity of mica increases, both parameters $\beta$ and $\gamma$ increase.

(2) As the size of mica increases, $\beta$ decreases and $\gamma$ increases.

(3) As the quantity of talc increases, $\beta$ decreases abruptly and $\gamma$ increases gradually. 
Table 2 shows a list of the estimated model parameters for the foundation IKD-0 IKD-59 with different material compositions. Thus, a variety of skin surface with different make-up foundations is described by the Cook-Torrance model with a small number of parameters.

Table 2. Composition and model parameters of a human hand with different foundations

\begin{tabular}{llccc}
\hline Samples & $\begin{array}{l}\text { Composition } \\
(\mathrm{M}, \mathrm{T})\end{array}$ & $\beta$ & $\gamma$ & $n$ \\
\hline IKD-0 & $(0,59)$ & 0.431 & 0.249 & 1.90 \\
IKD-10 & $(10,49)$ & 0.426 & 0.249 & 1.90 \\
IKD-20 & $(20,39)$ & 0.485 & 0.220 & 1.90 \\
IKD-40 & $(40,19)$ & 0.570 & 0.191 & 1.90 \\
IKD-54 & $(54,5)$ & 0.744 & 0.170 & 1.90 \\
IKD-59 & $(59,0)$ & 0.736 & 0.180 & 1.90 \\
\hline
\end{tabular}

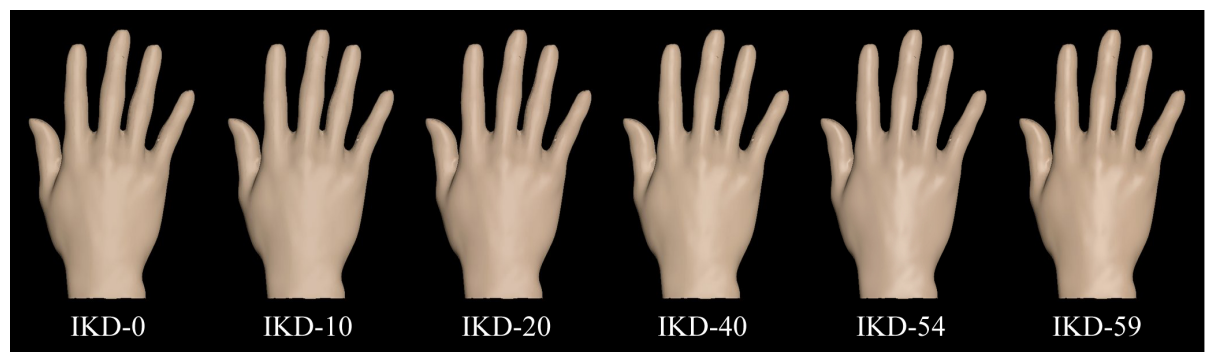

Fig. 6. Image rendering results for a human hand with different make-up foundations

For application to image rendering, we render color images of the skin surface of a human hand by using the present model fitting results. The 3D shape of the human hand was acquired separately by using a laser range finder system. Figure 6 demonstrates the image rendering results of the $3 \mathrm{D}$ skin surface with different make-up foundations. A ray-tracing algorithm was used for rendering realistic images, which performed wavelength-based color calculation precisely. Only the Cook-Torrance model was used for spectral reflectance computation of IKD-0 - IKD-59. We assume that the light source is D65 and the illumination direction is the normal direction to the hand.

In the rendered images, the appearance changes such that the gloss of skin surface increases with the quantity of mica. These rendered images show the feasibility of the model-based approach. A detailed comparison between spectral reflectance curves such as Fig. 5, however, suggests that there is a certain discrepancy between the measured reflectances and the estimated ones by the model. The similar discrepancy occurs for all the other samples.

\section{PCA-Based Analysis of Spectral Reflectance}

Let us consider another approach to describing spectral reflectance of the skin surface with make-up foundation. The PCA is effective for statistical analysis of the complicated spectral curves of the skin surface reflectance. 
First, we have to know the basic reflection property of the skin surface. In the previous report [2], we showed that the skin surface could be described by the standard dichromatic reflection model [6]. The standard model assumes that the surface reflection consists of two additive components, the body (diffuse) reflection and the interface (specular) reflection, which is independent of wavelength. The spectral reflectance (radiance factor) $Y\left(\theta_{i}, \theta_{r}, \lambda\right)$ of the skin surface is a function of the wavelength and the geometric parameters of incidence angle $\theta_{i}$ and viewing angle $\theta_{r}$. Therefore the reflectance is expressed in a linear combination of the diffuse reflectance $S(\lambda)$ and the constant reflectance as

$$
Y\left(\theta_{i}, \theta_{r}, \lambda\right)=C_{1}\left(\theta_{i}, \theta_{r}\right) S(\lambda)+C_{2}\left(\theta_{i}, \theta_{r}\right),
$$

where the weights $C_{1}\left(\theta_{i}, \theta_{r}\right)$ and $C_{2}\left(\theta_{i}, \theta_{r}\right)$ are the geometric scale factors.

To confirm the adequacy of this model, the PCA was applied to the whole set of spectral reflectance curves observed under different geometries of $\theta_{i}$ and $\theta_{r}$ with an equal $5 \mathrm{~nm}$ interval in the range $400-700 \mathrm{~nm}$. A singular value decomposition (SVD) is used for the practical PCA computation of spectral reflectances. The SVD shows twodimensionality of the set of spectral reflectance curves. Therefore, all spectral reflectances of skin surface can be represented by only two principal-component vectors $\mathbf{u}_{1}$ and $\mathbf{u}_{2}$. Moreover, $\mathbf{u}_{1}$ and $\mathbf{u}_{2}$ can be fitted to a unit vector $\mathbf{i}$ using linear regression, that is, the constant reflectance is represented by the two components. By the above reason, we can conclude that the skin surface has the property of the standard dichromatic reflection.

Next, let us consider the estimation of spectral reflectances for various angles of incidence and viewing without observation. Note that the observed spectral reflectances from the skin surface are described using the two components of the diffuse reflectance $S(\lambda)$ and the constant specular reflectance. Hence we expect that any unknown spectral reflectances are described in terms of the same components. Then the reflectances can be estimated by the following function with two parameters,

$$
Y\left(\theta_{i}, \theta_{r}, \lambda\right)=\hat{C}_{1}\left(\theta_{i}, \theta_{r}\right) S(\lambda)+\hat{C}_{2}\left(\theta_{i}, \theta_{r}\right),
$$

where $\hat{C}_{1}\left(\theta_{i}, \theta_{r}\right)$ and $\hat{C}_{2}\left(\theta_{i}, \theta_{r}\right)$ denote the estimates of the weighting coefficients on a pair of angles $\left(\theta_{i}, \theta_{r}\right)$.

In order to develop the estimation procedure, we analyze the weighting coefficients $C_{1}\left(\theta_{i}, \theta_{r}\right)$ and $C_{2}\left(\theta_{i}, \theta_{r}\right)$ based on the observed data. Again the SVD is applied to the data set of those weighting coefficients. When we consider an approximate representation of the weighting coefficients in terms of several principal components, the performance index of the chosen principle components is given by the percent variance $P(K)=\sum_{i=1}^{K} \mu_{i}^{2} / \sum_{i=1}^{n} \mu_{i}^{2}$. The performance indices are $P(2)=0.994$ for the first two components and $P(3)=0.996$ for the first three components in both coefficient data $C_{1}\left(\theta_{i}, \theta_{r}\right)$ and $C_{2}\left(\theta_{i}, \theta_{r}\right)$ from IKD-59. Then, the weighting coefficients can be decomposed into two basis functions with a single parameter as 


$$
C_{1}\left(\theta_{i}, \theta_{r}\right)=\sum_{j=1}^{K} w_{1 j}\left(\theta_{i}\right) v_{1 j}\left(\theta_{r}\right), \quad C_{2}\left(\theta_{i}, \theta_{r}\right)=\sum_{j=1}^{K} w_{2 j}\left(\theta_{i}\right) v_{2 j}\left(\theta_{r}\right),(K=2 \text { or } 3)
$$

where $\left(v_{1 j}\right)$ and $\left(v_{2 j}\right)$ are two sets of principal components as a function of viewing angle $\theta_{r}$, and $\left(w_{1 j}\right)$ and $\left(w_{2 j}\right)$ are two sets of the corresponding weights to those principal components, which are a function of incidence angle $\theta_{i} \cdot \hat{w}$ is the principal components and $v$ is the weights determined by interpolating the coefficients at observation points.

The performance values $P(2)$ and $P(3)$ are close each other. We examine the accuracy in the two cases for describing the surface-spectral reflectances under all observation conditions. Figure 7 depicts the root-mean squared errors (RMSE) of the reflectance approximation for $K=2,3$. In the case of $K=2$, although the absolute error of overall fitting is relatively small, noticeable errors occur at the incident angles of around 0,40 , and 60 degrees. In particular, it should be emphasized that the errors at the incident and viewing angles of around 0 degree deteriorate seriously the image rendering results of $3 \mathrm{D}$ objects. We find that $K=3$ improves much to express the surface-spectral reflectances by only one additional component.

Therefore the estimation of $\hat{C}_{1}\left(\theta_{i}, \theta_{r}\right)$ and $\hat{C}_{2}\left(\theta_{i}, \theta_{r}\right)$ for any unknown reflectance can be reduced into a simple form

$$
\begin{aligned}
& \hat{C}_{1}\left(\theta_{i}, \theta_{r}\right)=\hat{w}_{11}\left(\theta_{i}\right) v_{11}\left(\theta_{r}\right)+\hat{w}_{12}\left(\theta_{i}\right) v_{12}\left(\theta_{r}\right)+\hat{w}_{13}\left(\theta_{i}\right) v_{13}\left(\theta_{r}\right) \\
& \hat{C}_{2}\left(\theta_{i}, \theta_{r}\right)=\hat{w}_{21}\left(\theta_{i}\right) v_{21}\left(\theta_{r}\right)+\hat{w}_{22}\left(\theta_{i}\right) v_{22}\left(\theta_{r}\right)+\hat{w}_{23}\left(\theta_{i}\right) v_{23}\left(\theta_{r}\right)
\end{aligned},
$$

where $\hat{w}_{i j}\left(\theta_{i}\right)(i=1,2 ; j=1,2,3)$ are determined by interpolating the coefficients at observation points such as $w_{i j}(0), w_{i j}(5), \ldots, w_{i j}(60)$. Thus, the spectral reflectance of the skin surface at arbitrary angular conditions is generated using the diffuse spectral reflectance $S(\lambda)$, the principal component $v_{i j}\left(\theta_{r}\right)(i=1,2 ; j=1,2,3)$, and three pairs of weights $\hat{w}_{i j}\left(\theta_{i}\right)(i=1,2 ; j=1,2,3)$. Note that these basis data are all onedimensional.

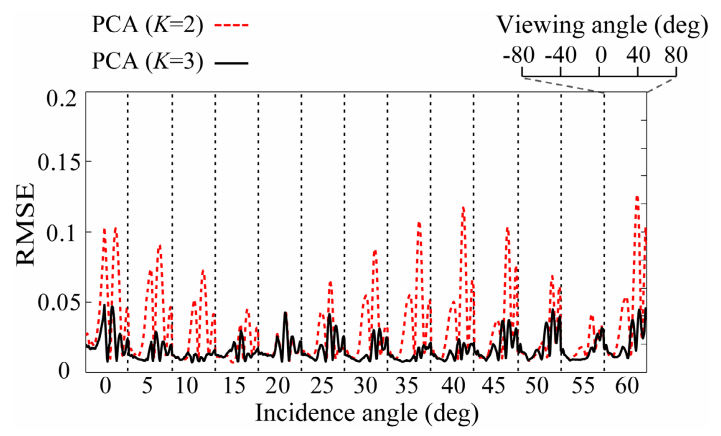

Fig. 7. RMSE in IKD-54 reflectance approximation for $K=2,3$ 


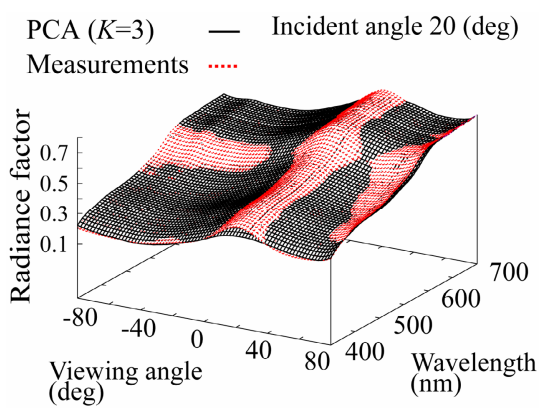

(a)

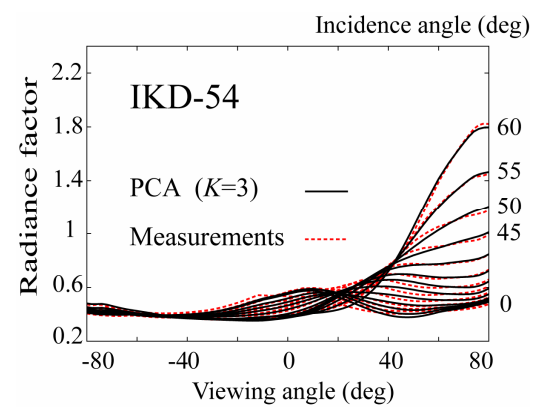

(b)

Fig. 8. Estimation results of surface-spectral reflectances for IKD-54. (a) 3D view of spectral reflectances at $\theta_{i}=20$, (b) Average reflectances as a function of viewing angle.

Figure 8 shows the estimation results to the sample IKD-54, where solid curves indicates the reflectances by the proposed method, and broken curves indicate the original measurements. We should note that the surface spectral reflectances of the skin with make-up foundation are recovered with sufficient accuracy.

\section{Performance Comparisons and Applications}

A comparison between Fig. 8 by the PCA method and Fig. 5 by the Cook-Torrance model suggests clearly that the estimated surface-spectral reflectances with $K=3$ are almost coincident with the measurements at all angles. The estimated spectral curves represent accurately the whole features of skin reflectance, including not only reflectance hump at around the vertical viewing angle, but also back-scattering at around 70 degrees, and increasing reflectance at around 70 degrees.

Figure 9 shows the typical estimation results of surface-spectral reflectance of IKD-54 at the incidence of 20 degrees. The estimated reflectance by the PCA method is more closely coincident with the measurements at all angles, while clear discrepancy occurs for the Cook-Torrance model at large viewing angles. Figure 10 summarizes the RMSE of both methods for IKD-54. The solid mesh indicates the estimation results by the Cook-Torrance method and the broken mesh indicates the estimates by the PCA method. The PCA method with $K=3$ provides much better performance than the Cook-Torrance model method. Note that the Cook-Torrance method has large discrepancy at the two extreme angles of the viewing range [-70, 70].

Figure 11 demonstrates the rendered images of a human hand with the foundation IKD-54 by using both methods. Again the wavelength-based ray-tracing algorithm was used for rendering the images. The illumination is D65 from the direction of 45 degrees to the surface normal. It should be noted that, although the rendered images represent a realistic appearance of the human hand, the image by the PCA method is sufficiently close to the real one. It looks more natural and warm atmosphere like our skins. The same results were obtained for all foundations IKD-0 - IKD-50 with different material compositions. 


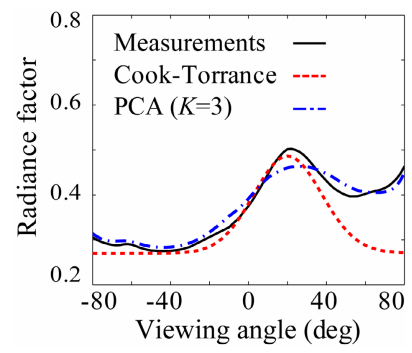

Fig. 9. Reflectance estimates for IKD-54 as a function of viewing angle

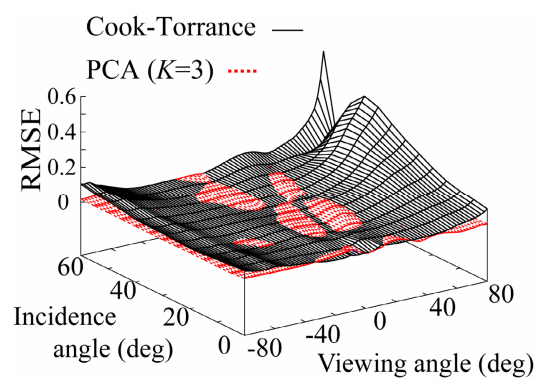

Fig. 10. RMSE in IKD-54 reflectance estimates

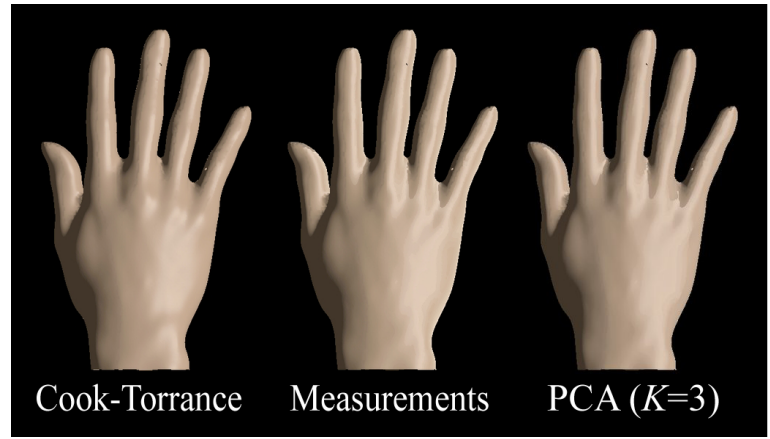

Fig. 11. Image rendering results of a human hand with make-up foundation IKD-54

\section{Conclusions}

This paper has described the detailed analysis of the spectral reflection properties of skin surface with make-up foundation, based on two approaches of a physical model using the Cook-Torrance model and a statistical approach using the PCA.

First, we showed how the surface-spectral reflectances changed with the observation conditions of light incidence and viewing, and also the material compositions. Second, the Cook-Torrance model was useful for describing the complicated reflectance curves by a small number of parameters, and rendering images of 3D object surfaces. We showed that parameter $\beta$ increased as the quantity of mica increased. However, the model did not have sufficient accuracy for describing the surface reflection under some geometry conditions. Third, the PCA of the observed spectral reflectances suggested that all skin surfaces satisfied the property of the standard dichromatic reflection. Then the observed reflectances were represented by two spectral components of a diffuse reflectance and constant reflectance. The spectral estimation was reduced to a simple computation using the diffuse reflectance, some principal components, and the weighting coefficients. The PCA method could describe the surface reflection properties with foundation with sufficient accuracy. Finally, the feasibility was examined in experiments. It was shown that the PCA method 
could provide reliable estimates of the surface-spectral reflectance for the foundation skin from a global point of view, compared with the Cook-Torrance model.

The investigation into the physical meanings and properties of the principal components and weights remains as future works.

\section{References}

1. Boré, P.: Cosmetic Analysis: Selective Methods and Techniques. Marcel Dekker, New York (1985)

2. Tominaga, S., Moriuchi, Y.: PCA-based reflectance analysis/synthesis of cosmetic foundation. In: CIC 16, pp. 195-200 (2008)

3. Phong, B.T.: Illumination for computer-generated pictures. Comm. ACM 18(6), 311-317 (1975)

4. Cook, R., Torrance, K.: A reflection model for computer graphics. In: Proc. SIGGRAPH 1981, vol. 15(3), pp. 307-316 (1981)

5. Torrance, K.E., Sparrow, E.M.: Theory for off-specular reflection from roughened surfaces. J. of Optical Society of America 57, 1105-1114 (1967)

6. Born, M., Wolf, E.: Principles of Optics, pp. 36-51. Pergamon Press, Oxford (1987) 